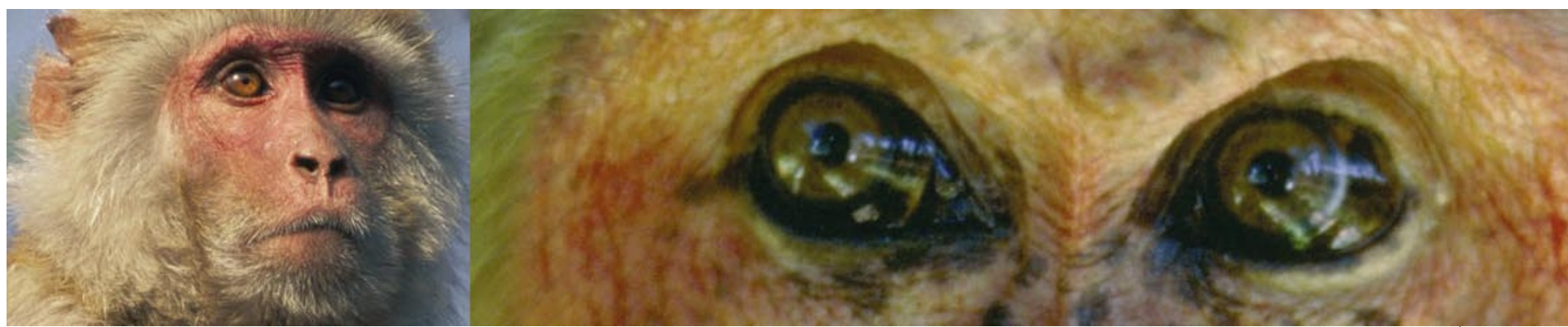

\title{
Decisions, decisions...
}

\author{
By recording the electrical activity of individual neurons in monkeys, \\ neuroscientists are beginning to understand how the brain makes simple \\ decisions. Bas Kast considers the links between perception and action.
}

\author{
"As soon as questions of will or decision \\ or reason or choice of action arise, \\ human science is at a loss."
}

W hen the US linguist and political scientist Noam Chomsky uttered these words in a television interview in 1978, they had the ring of truth. Researchers were starting to get to grips with how the brain perceives events and how it acts upon them. But they had no real understanding of the decision-making processes that link perception and action.

Since then, neuroscientists interested in vision and other sensory systems have studied the brain areas involved in various levels of information processing. Meanwhile, other researchers have unravelled the mechanisms behind complex body movements. "The groups have met at the missing link the brain locus of decision," says William Newsome, a neuroscientist at Stanford University in California. And over the past few years, Newsome's group, plus a handful of others, has started to reveal the neural mechanisms that underlie simple decision-making.

By stripping decision-making down to its bare essentials, Newsome hopes to reveal the basic mechanisms at work in the brain. "This terrain is sufficiently complex even for the simplest cases," he says.

In a landmark 1996 experiment $^{1}$, for instance, Newsome's group presented monkeys with a set of moving dots, a fraction of which where moving in the same direction. The monkeys were trained, using rewards of a drink, to work out which way most of the dots were moving and make a quick, jerklike, eye movement in the same direction. The task was easy when all the dots were moving together. But in some trials only a few dots moved coherently in one direction, while the others moved around randomly.
The decision became more difficult as the proportion of coherently moving dots decreased. And in the extreme condition, where all dots were moving at random, the monkeys were forced to guess.

\section{Spot the difference}

To discover how each monkey used the moving dots to make a decision, Newsome recorded signals - voltage pulses - sent by individual neurons in the monkey's brain while it was working on the task.

Neuroscientists were already familiar with neurons involved in visual processing that are 'tuned' to motion in a particular direction these cells only fire when they see objects moving in their preferred direction. Similar cells in motor areas fire when the monkey moves its eyes or limbs in a particular direction.

Together with Michael Shadlen, Newsome recorded the activity of cells in the lateral intraparietal cortex (LIP), a brain region linking areas involved in visual processing to those that control eye movement. They identified LIP cells that responded either to dots moving to the left or to the right. But unlike the tuned cells in visual processing areas, the LIP cells also fired when the monkeys were about to make a guess about the direction in which randomly moving dots were going. Clearly, they were not simply responding to the pattern. "After a while, we noticed that we could predict the direction of the monkey's eye movement by monitoring the activity of a single cell alone," says Newsome.

These cells could merely have been

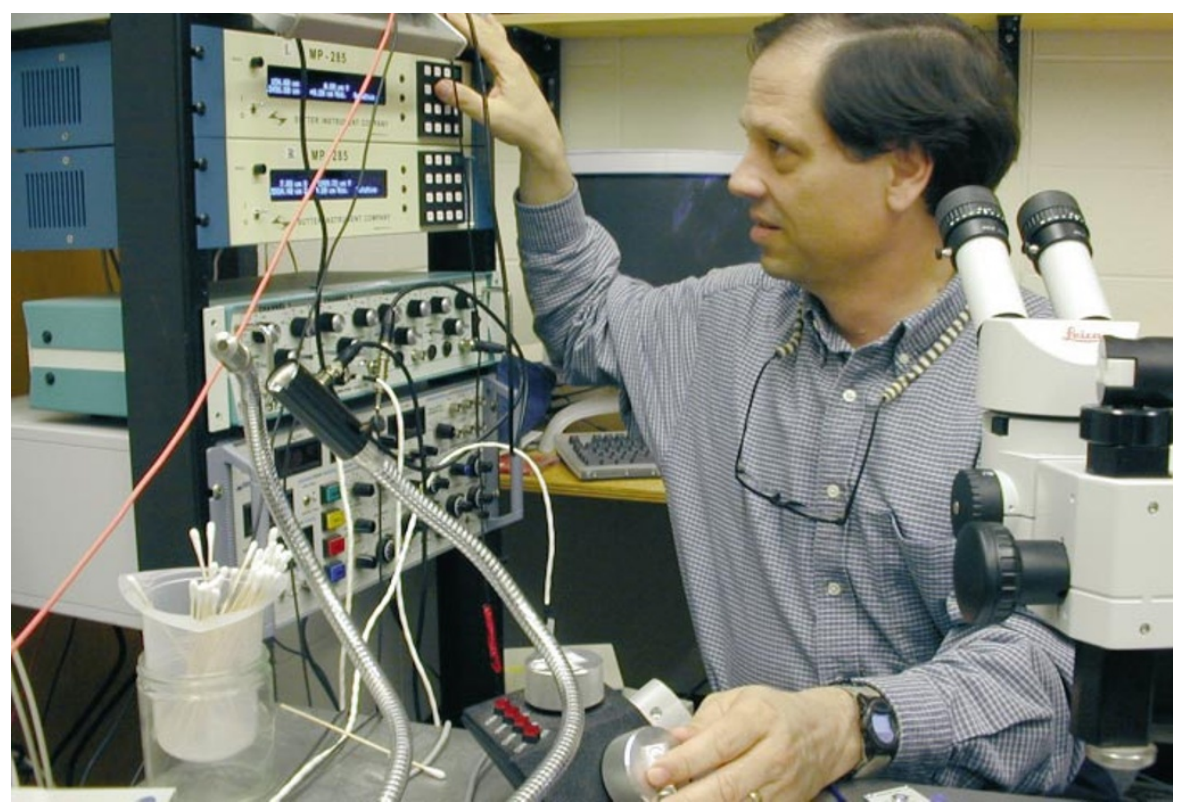

First choice: William Newsome has identified brain cells that appear to make simple decisions. 


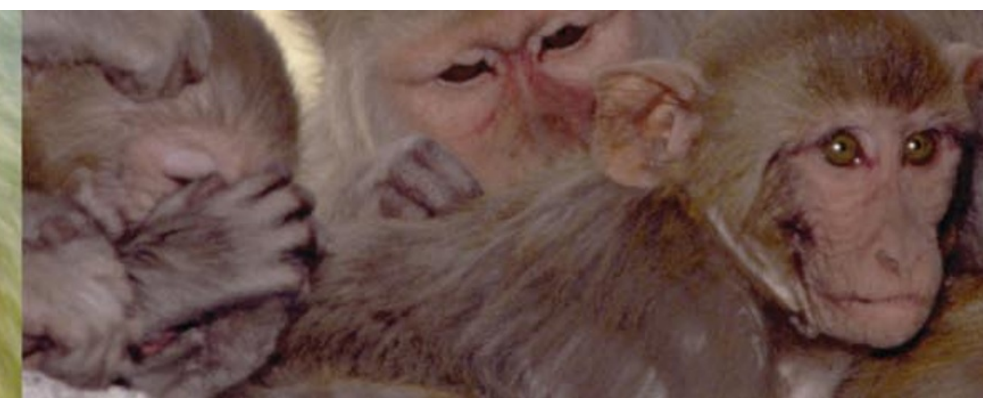

controlling the monkeys' eye movements. But Newsome and Shadlen noticed that the cells fired off more pulses when the decision was easy. As the number of coherently moving dots decreased and the choice got harder, tuned cells responded with fewer pulses, even though the monkey was still making a decision to move its eyes. The cells seemed both to respond to the difficulty of the pattern and to influence the monkey's movement, something a purely visual or motor cell could not have done. "Rather than reflecting either sensory or motor information, these cells seemed to integrate both," says Newsome.

Newsome believes that his cells are 'decision neurons', and other groups are now building from his work. After moving to the University of Washington in Seattle, Shadlen teamed up with neuroscientist Jong-Nam Kim to try to explain how such cells make the choice of whether to tell the motor system to make an eye movement.

\section{Under observation}

In 1999, Kim and Shadlen identified a similar population of decision cells in a region near the front of the brain called the prefrontal cortex ${ }^{2}$. These neurons are connected to a brain region involved in visual processing called the middle temporal (MT) area, which contains cells that respond to either leftward or rightward movement. Kim and Shadlen suggested that the decision neurons make their choices by monitoring the activity of the direction-tuned MT cells, and then send instructions to cells in the superior colliculus that control movement.

If a monkey looks at a pattern in which most of the dots are moving to the right, neurons in the MT tuned to rightward movement will become active. Kim and Shadlen reasoned that decision neurons observing this burst of activity, together with a corresponding lack of activity in left-tuned MT neurons, will activate the motor system to jerk the eyes to the right so that the monkey receives its reward.

Shadlen and Kim's model has yet to be confirmed by recording from several cells simultaneously. But it does explain why decision neurons fire less as the number of coherently moving dots decreases, as the difference between the firing rates of the left- and right-tuned MT neurons will become less marked.

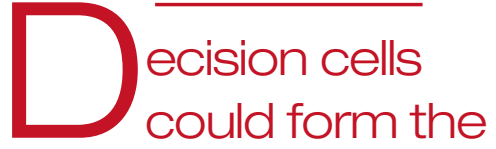

\section{basis for a 'central executive' in the brain.}

The model also explains why monkeys sometimes make the wrong decision. Like other neurons, MT cells are inherently 'noisy' - the number of times they fire varies from trial to trial, even when the monkey sees exactly the same stimulus. This means that a burst of firing by left-tuned cells could, by chance, occasionally overwhelm a low signal from right-tuned cells, even if the majority of dots are moving to the right. But Kim and Shadlen's model says that it should still be possible to predict the direction of the monkey's eye movement from the firing of decision cells. This is exactly what Newsome and Shadlen had found previously ${ }^{1}$.

The study of decision neurons has now extended beyond vision. Ranulfo Romo, a neurophysiologist at the National Autonomous University of Mexico, has identified neurons involved in choices based on tactile stimuli ${ }^{3}$. Similar cells for decisions based on smell or sound may also exist.

\section{Executive control}

But Romo and some other neuroscientists go one step further. They propose that the brain's frontal lobe contains decisionmaking cells that are independent of any of the senses. Such cells could form the basis for a 'central executive' in the brain - an area in overall control of decisions. Central executives are a feature of many psychological models of decision-making and the idea gels naturally with our feeling of being in charge of our bodies and minds.

But Newsome and other experts question whether such a central decision-maker will ever be found. Complex decisions, Newsome suggests, are made in a distributed way. "The locus of decision lies in the pattern of connections between sensory inputs and motor outputs. Just as we cannot pinpoint the self in the brain, we cannot determine the decision-maker," he says. "But opinion is highly divided. At the end of the day, we are all just following our intuitions on this issue."
Determining who is right would involve training monkeys to make decisions based on information from more than one of their senses. And so far, such sophisticated experiments have proved beyond the reach of even the most skilled neurophysiologists.

Other neuroscientists believe that the new ideas about decision neurons can be extended to processes that seem not to involve conscious control. Confronted by an overwhelming mass of visual information, areas of the brain involved in visual processing constantly make 'decisions' that simplify what we see. Researchers can investigate how the brain does this by using stimuli that make the decisions difficult. The Necker cube illusion is one example. Our perception of the cube seems to jump between two states - in the version shown overleaf, the green dot can be either on the front or back face of the cube. Unable to decide which is correct, the brain switches back and forth between the two.

Neuroscientists David Leopold and Nikos Logothetis of the Max Planck Institute for Biological Cybernetics in Tübingen, Germany, have used a similar phenomenon to study perceptual decisions. Known as binocular rivalry, the effect can be induced by projecting a different pattern into each of a subject's eyes. Even though both patterns are present at the same time, human volunteers report seeing each one alternately ${ }^{4}$.

Leopold and Logothetis have also measured the activity of neurons in visual areas of brains in monkeys that were viewing different patterns through each eye - one

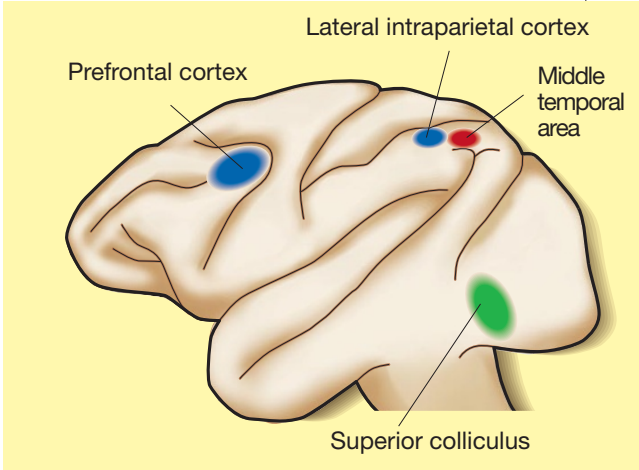

Cells that control eye movement (green) are given instructions by decision-making cells (blue), apparently based on the latter's observations of cells in the visual-processing region (red). 


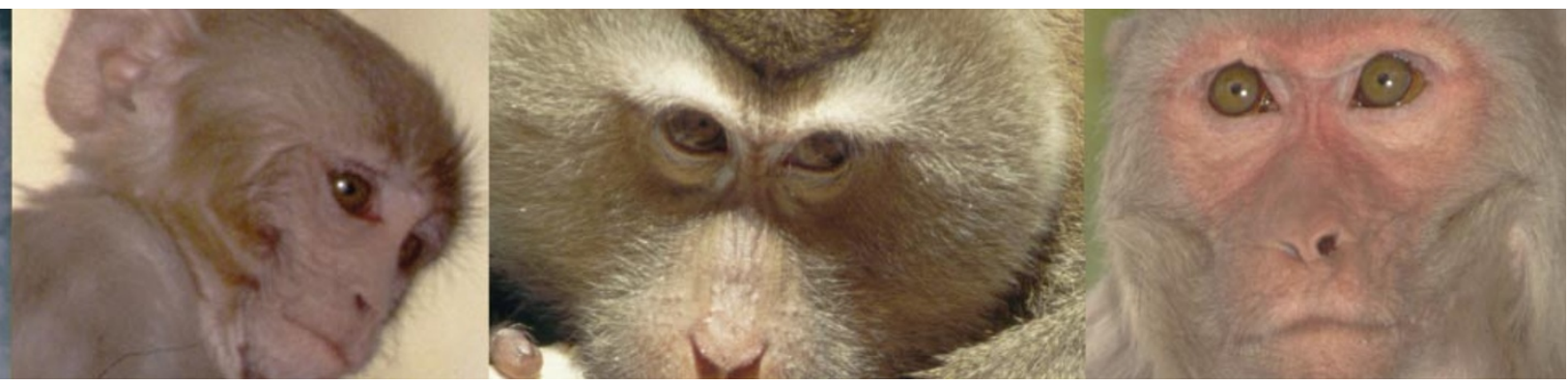

moving upwards, the other downwards ${ }^{4}$. The monkeys had previously been trained to pull a different lever in response to each pattern, so by recording the animals' responses, the researchers could tell which pattern their brain was perceiving at any one time.

As expected, Leopold and Logothetis identified visual-processing cells tuned exclusively to either upward or downward motion. But the activity of other cells in the monkey's visual processing areas depended on what pattern the monkeys had actually perceived. Some downward-tuned cells, for example, fired only when the monkey pulled on the 'downwards' lever. Leopold and Logothetis propose that these cells are communicating with 'planning' brain areas that are involved in deciding which of the images is actually seen. The mechanism, they argue, is similar to that investigated by Newsome.

\section{Perceptive planning}

Other evidence hints that the frontal cortex may be the planning area. The perception of similar illusions can be impaired by damage to the prefrontal cortex ${ }^{5}$, and imaging studies of the human brain have shown that the frontal lobe is active during the perceptual shifts experienced in binocular rivalry ${ }^{6}$. "It doesn't mean that the frontal lobe makes this decision," stresses Leopold, "but deciding to see one image or another, if deciding indeed is the right word to use, might be similar to the decision of moving an eye to the left or the right. The obvious difference is that the output of this behaviour is not a

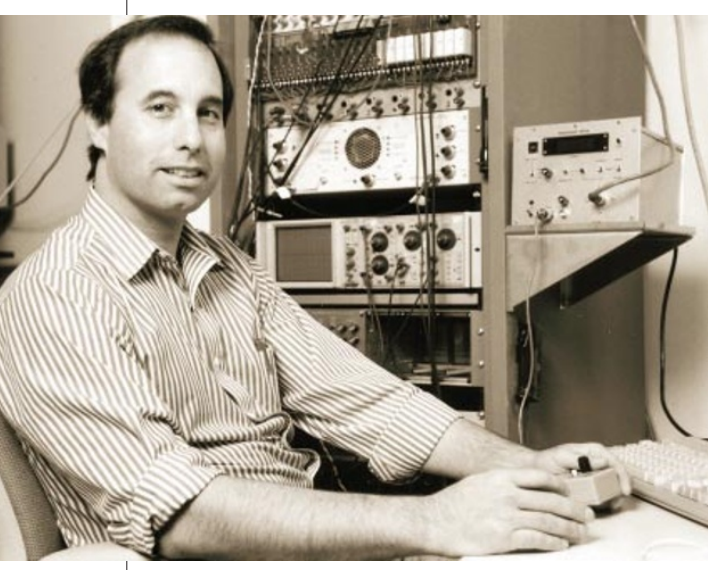

Look and learn: Michael Shadlen is studying how decision cells make their choices. motor response, but a perceptual change."

Most people intuitively feel that 'deciding' what to see and making conscious decisions are different processes. But Leopold and Logothetis dispute this, pointing out that we can exercise some conscious control over perceptual decisions - try holding one version of the Necker cube steady, for example. And many of the decisions that we make voluntarily, such as braking while driving, become such second nature that they are essentially unconscious actions.

Christian Keysers, a neuroscientist at the University of Parma in Italy, agrees. "Perceptual decisions are probably the result of a simple, winner-takes-all competition between the neural activity representing alternative perceptions," he says. "It's likely that an equivalent process of neural competition also applies when decisions are not about perceptions but between motor outputs."

But even if the decisions studied by Leopold and Logothetis are equivalent to those investigated by Newsome, both are very different from the endless decisions that fill our waking hours. For one thing, the monkeys studied by neuroscientists are responding to simple stimuli, whereas most of our conscious decisions take account of accumulated knowledge or experience.

\section{Remembrance of things past}

Michael Platt of Duke University in Durham, North Carolina, and Paul Glimcher of New York University have attempted to address this objection. They trained monkeys to focus on a light and, when the light changed colour, to make an eye movement. In one block of 100 trials, an eye movement to the right would be rewarded with more than twice as much orange juice as one to left. In other blocks, the rewards were reversed. Over the course of each block of 100 trials, the monkeys learnt which direction brought the biggest reward and consistently moved their eyes in that direction. Lacking a stimulus to tell them which way to go, the monkeys based their decisions on the reward associated with previous trials.

Platt and Glimcher recorded data from LIP neurons tuned to eye movements and found that right-tuned cells fired at higher frequencies when the monkey could expect a greater reward by going to the right ${ }^{7}$. This knowledge had to come from the monkey's

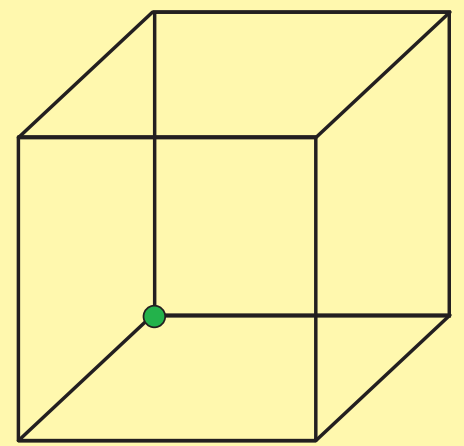

Boxed in: the Necker cube illusion confuses our brain as it cannot work out whether the green dot is at the front or the rear of the object.

memory of previous trials, suggesting that the LIP cells were using this memory to help the monkey make its decision. "This is the kind of problem an animal has to solve all the time," says Platt. "Most of our choices are guided by the pay-offs associated with different actions in the past." He is now searching for the brain region that signals the size of the reward to the LIP neurons.

For the different groups studying the neurophysiology of decision-making, expanding their work to incorporate more complex decisions is the next big step. Even the most sophisticated of the decisions studied in current experiments are still a long way from simple everyday decisions such as what to eat for dinner. The big decisions in our lives - such as whether to take a new job or to get married - are still as inaccessible as Chomsky said they were in 1978.

Newsome does not deny this. But he believes that starting simple provides the best means to investigate the processes that underlie more complex decisions. "Maybe the kinds of decisions we presently study are not what we typically regard as the most interesting decisions that we make every day," he concedes. "But, hey, the neuroscientist has to start somewhere."

Bas Kast writes for Der Tagesspiegel in Berlin.

1. Shadlen, M. N. \& Newsome, W. T. Proc. Natl Acad. Sci. USA 93, 628-633 (1996)

2. Kim, J.-N. \& Shadlen, M. N. Nature Neurosci. 2, 176-185 (1999). 3. Romo, R. \& Salinas, E. Annu. Rev. Neurosci. 24, 107-137 (2001).

4. Leopold, D. A. \& Logothetis, N. K. Trends Cogn. Sci. 3, 254-264 (1999).

5. Ricci, C. \& Blundo, C. Neuropsychologia 28, 1163-1173 (1990)

6. Lumer, E. D., Friston, K. J. \& Rees, G. Science 280, 1930-1934 (1998).

Platt, M. L. \& Glimcher, P. W. Nature 400, 233-238 (1999). 